\title{
Distribución, bioecología y provisión de bienes y servicios ecosistémicos de Prosopis alba en Argentina
}

\author{
Maria Laura Fontana ${ }^{1,2, *}$; Víctor Ramón Pérez ${ }^{3}$; Claudia Verónica Luna ${ }^{1,4}$
}

${ }^{1}$ Facultad de Ciencias Agrarias - Universidad Nacional del Nordeste // INTA EEA Corrientes; ${ }^{2}$ INTA Estación Experimental Agropecuaria Sáenz Peña, Presidencia Roque Sáenz Peña, Chaco, Argentina;

3Universidad Nacional de Formosa - Facultad de Recursos Naturales - Cátedra de Silvicultura, Formosa, Argentina; ${ }^{4}$ CONICET - Instituto de Botánica del Nordeste (IBONE), Corrientes, Argentina;

*ma.la.fo@hotmail.com

Fontana Maria Laura; Víctor Ramón Pérez; Claudia Verónica Luna (2020). Distribución, bioecología y provisión de bienes y servicios ecosistémicos de Prosopis alba en Argentina. Rev. Fac. Agron. Vol 119 (2): 1-11. https://doi.org/10.24215/16699513e050

\begin{abstract}
Prosopis alba (Grisebach), vulgarmente conocido como algarrobo blanco en Argentina, es una planta leñosa con amplia distribución en Sudamérica: Argentina, Uruguay, Paraguay, Brasil, Bolivia, Chile y Perú. Son árboles pioneros, heliófilos, adaptados a condiciones de climas áridos y semiáridos con suelos salinos. Se la define como una especie multipropósito de gran importancia regional debido a la diversidad de usos tanto madereros como alimenticios, forrajeros y medicinales; brinda servicios ecosistémicos y mejora los ambientes, teniendo potencial para restaurar áreas degradadas y catalizar el desarrollo de nuevos sistemas de producción. El objetivo de esta revisión es recuperar y trascender el conocimiento acumulado sobre su taxonomía, caracteres botánicos, distribución y bioecología, así como los bienes y servicios que proporciona esta especie forestal de gran interés para Argentina.
\end{abstract}

Palabras clave: algarrobo blanco; algarroba; bienes y servicios forestales; productos forestales no madereros; productos forestales madereros

Fontana María Laura; Víctor Ramón Pérez; Claudia Verónica Luna (2020). Distribution, bio-ecology and ecosystem goods and services supply of Prosopis alba in Argentina. Rev. Fac. Agron. Vol 119 (2): 1-11. https://doi.org/10.24215/16699513e050

Prosopis alba (Grisebach), commonly known as "algarrobo blanco" in Argentina is a woody plant with wide distribution in South America- Argentina, Uruguay, Paraguay, Brazil, Bolivia, Chile and Peru. It is a pioneer tree, heliophileous, adapted to arid and semi-arid climates, and to saline soils. It is defined as a multipurpose species of high regional importance, since it is a provider of timber, edible fruits, forage and medicine. It also provides ecosystem services and improves the environment having potential to restore degraded ecosystems and catalyze the development of new production systems. The aim of this revision is to recover and transcend the accumulated knowledge about its taxonomy, botanical characteristics, distribution and bio-ecology, as well as the goods and services provided by this forest tree species of great interest for Argentina.

Keywords: algarrobo blanco; algarroba; forest goods and services; non-timber forest products; timber forest products

https://revistas.unlp.edu.ar/revagro

Recibido: 02/08/2019

Aceptado: $11 / 02 / 2020$

Disponible on line: 07/12/2020

ISSN 0041-8676 - ISSN (on line) 1669-9513, Facultad de Ciencias Agrarias y Forestales, UNLP, Argentina 


\section{INTRODUCCIÓN}

En el continente americano el género Prosopis se ubica entre los $37^{\circ}$ de latitud norte y los $48^{\circ}$ de latitud sur, desde el sudoeste de Estados Unidos a lo largo de los Andes hasta Chile central y Argentina, donde habita la mayor cantidad de especies (27 especies y 19 variedades) (Palacios \& Brizuela, 2005). La utilidad ecológica y social de los Prosopis varía de una especie a otra: así como varias especies son malezas en partes de su área de distribución nativa (Pasiecznik et al., 2001), en Argentina, Prosopis alba resulta ser de gran importancia económica por la provisión de materia prima (López, 2005) y se la menciona como la principal especie nativa empleada en la industria del mueble (Michela et al., 2015). En este contexto, el objetivo de esta revisión fue compilar información relacionada a la taxonomía, caracteres botánicos, distribución y bioecología, así como los bienes y servicios que proporciona el algarrobo blanco, una especie forestal de interés para Argentina. Además de la consulta y valoración crítica de publicaciones científicas y tecnológicas nacionales e internacionales, también se revisaron 7 tesis de grado y posgrado, estadísticas nacionales y provinciales, y se consideraron los marcos regulatorios de las leyes forestales en vigencia para analizar la producción sustentable del recurso.

\section{TAXONOMÍA Y GENÉTICA}

Prosopis alba (Grisebach), vulgarmente conocido como algarrobo blanco en Argentina, pertenece al clado Mimosoid, subfamilia Caesalpinioideae de la familia Fabaceae (Legume Phylogeny Working Group, 2017). Se trata de una especie diploide $(2 n=28)$ incluida en la serie Chilensis de la sección Algarobia (Burkart, 1976), sección que contiene a las especies más importantes desde el punto de vista económico y ecológico (Roser et al., 2013). Se reconocen dos variedades: $P$. alba var. alba y $P$. alba var. panta. Esta última, según estudios moleculares, está más estrechamente relacionada con otras especies de Prosopis que con P. alba, lo que sugiere una considerable variación genética (Pasiecznik et al., 2004).

Karlin (1988) y Gómez-Sosa \& Palacios (1994) mencionan que la taxonomía del género Prosopis resulta compleja debido a la gran variación fenotípica dentro de las especies y por la frecuente hibridación natural que ocurre entre ellas. Originalmente Burkart (1976) dividió al género en 5 secciones, algunas de ellas subdivididas en series (separadas por diferencias vegetativas). La sección Algarobia habitaría las zonas más cálidas y secas de América y agruparía a individuos de porte arbóreo o arbustivo, con espinas axilares uninodales, solitarias o frecuentemente en pares (Barros, 2010). La información existente señala que las 6 series de esta sección (Sericanthae, Pallidae, Ruscifoliae, Denudans, Humiles y Chilenses) carecen de un sustento isoenzimático por lo cual no conformarían grupos naturales (Bessega et al., 2005) y la relación entre las especies aún está en debate (Pasiecznik et al., 2001). Sumada a esta complejidad para esclarecer la correcta ordenación, existen registros de hibridación entre especies de la serie Chilenses en zonas simpátricas (Hunziker et al., 1986).

La aplicación de taxonomía numérica y el análisis molecular develarían algunas cuestiones, como las que lograron revelar Verga et al. (2009) aplicando dichas técnicas en poblaciones naturales de algarrobos de la serie Chilensis localizados en el Parque Chaqueño y el Espinal Norte (Argentina). Estos autores comprobaron la existencia de, al menos, dos formotipos de Prosopis alba en la región chaqueña que se diferencian con cierta facilidad. Ello permitiría pensar que ciertas diferencias morfológicas (sin diferencias genéticas significativas) asignadas al rango de especie o variedad por Burkart, podrían corresponder en realidad al nivel de subespecies o razas geográficas.

\section{CARACTERES BOTÁNICOS}

Prosopis alba se encuentra entre las once especies del género que son endémicas de Argentina y se la considera una de las maderas nativas de mayor uso (Verga, 2005). Es una planta leñosa de estructura tortuosa que alcanza 12 a $15 \mathrm{~m}$ de altura y $1,5 \mathrm{~m}$ de diámetro de fuste. Sus hojas son bipinnaticompuestas con 1 a 3 yugas, glabras, pecioladas, con el raquis de 0,5 a $8 \mathrm{~cm}$ de longitud. Las pinnas presentan 25-50 pares de foliolulos lineales rectos o levemente encorvados, agudos, subsésiles y de base asimétrica que miden de $0,5-1,7 \mathrm{~cm}$ de longitud por $1-2 \mathrm{~mm}$ de lado. Son subcoriáceos, glabros, con una distancia entre folíolos de 1,5 a $6 \mathrm{~mm}$ (Galera, 2000).

Posee racimos cilíndricos amarillos, de 7 a $11 \mathrm{~cm}$ de longitud, con flores hermafroditas blanco-verdosas a amarillentas, pequeñas, subsésiles (Galera, 2000). Los frutos son vainas lineares, arqueadas o anulares, de 12 a $25 \mathrm{~cm}$ de largo; 1,2 a $1,8 \mathrm{~cm}$ de ancho y $0,5 \mathrm{~cm}$ de espesor, color amarillo paja, muy comprimidas, de bordes paralelos, con semillas que se identifican en las caras laterales y pulpa dulce; produce de 5 a $40 \mathrm{~kg}$ de frutos/año y fructifica entre diciembre y marzo. Las semillas (15 a 30/vaina) son de forma ovoidal, de 6 a 7 $\mathrm{mm}$ de largo, 4 a $5 \mathrm{~mm}$ de ancho, y de color castaño (Burkart, 1976; Felker, 1999). El endosperma es vítreo y asimétrico. Los cotiledones son de color amarillo claro, en forma de "s", con base asimétrica redondeada en la parte de la radícula, más pronunciada que en las otras especies (Galera, 2000).

Por otra parte, la caracterización morfológica de los algarrobos en las regiones fitogeográficas Chaqueña y Espinal norte de Argentina realizada por Verga et al. (2009) permitieron diferenciar tres morfotipos de $P$. alba var. alba empleando principalmente caracteres de hoja. En este sentido, $P$. alba santiagueño presenta hojas mayores, con mayor largo y ancho de foliólulo, mayor longitud de pecíolo y de pina y mayor distancia entre foliólulos, tiene menor número de foliólulos por pina y son raras las hojas con tres pares de pinas; la forma del foliólulo es más delgado y de ápice más aguzado que el morfotipo chaqueño. Entre los dos tipos de algarrobo blanco chaqueño la diferenciación es significativamente menor: chaqueño norte se caracteriza por poseer hojas con numerosos pares de foliólulos y muy cercanos entre sí y, chaqueño sur, es parecido al anterior, pero de foliólulos más anchos y un poco más separados. 
Las principales diferencias entre las variedades alba y panta son que los folíolos de la var. panta son más pubescentes y más largos que los de alba. Los frutos de panta son rectos, más oscuros, con más mesocarpo (pulpa) y sin bordes sobresalientes, a veces con manchas y de mayor tamaño. La variedad panta se encuentra representada por individuos escasos y dispersos, en virtud de que por sus excelentes características ha sido muy explotada, quedando pocos remanentes de ellos en el NO de la Provincia de Córdoba (Galera, 1995).

\section{DISTRIBUCIÓN Y BIOECOLOGÍA}

La especie posee una extensa área de distribución natural en Sudamérica: se encuentra en Argentina, Uruguay, Paraguay, en el pantanal de Brasil, sur de Bolivia y norte de Chile y Perú (Delvalle et al., 2003). En Argentina es muy abundante en la zona centro y norte del país, en las provincias fitogeográficas del Chaco, Espinal y del Monte (Ledesma et al., 2008); se la encuentra principalmente en las provincias de Córdoba, Santiago del Estero, La Rioja, Catamarca, Tucumán, Salta, Santa Fe, Chaco y Formosa (Venier et al., 2013). Existen tres "ecotipos" de algarrobos blancos diferenciados a nivel morfológico, principalmente a través de sus caracteres de hojas: $P$. alba "Santiagueño", $P$. alba "Chaqueño norte" y $P$. alba "Chaqueño sur" (Verga et al., 2009).

Ocupa el segundo estrato en los bosques altos de madera dura llegando a dominar cuando ocurre la eliminación de los quebrachos (Schinopsis spp., Aspidosperma quebracho blanco). Vive también en comunidades puras fuera del bosque y es común en las galerías de ríos, así como también cinturones alrededor de depresiones salinas suaves (Karlin, 1988). Está presente asimismo en las sabanas con suelos arenosos, siempre que la capa freática no esté a más de 15 metros de profundidad. Según Carnevale (1955) el algarrobo puede vivir en suelos con bajo tenor de humedad hasta el metro de profundidad pues el enorme desarrollo de su sistema radicular le permite la absorción de agua.

Se trata de una especie de árboles pioneros, heliófilos, adaptados a condiciones de climas áridos y semiáridos con suelos salinos y degradados. Se la considera rústica, con gran plasticidad y soporta condiciones extremas de humedad y temperatura (Di Marco, 2013). La ocurrencia de estreses tempranos (altas temperaturas, falta de agua y salinidad durante la germinación) no afectan en mayor grado el desarrollo de plántulas, característica ecológica que junto a las anteriores la califican como una potencial invasora (Killian, 2012).

Su distribución ocupa áreas con temperaturas extremas comprendidas entre los $48^{\circ} \mathrm{C}$ y los $-10^{\circ} \mathrm{C}$ y de 500 a $1200 \mathrm{~mm}$ de precipitación anual con concentración estival (Karlin et al., 1997). Donde las precipitaciones varían entre 500 y $600 \mathrm{~mm}$ se comporta como freatófita, encontrándose preferentemente a orillas de cañadas y ciénagas o a lo largo de ríos y arroyos. Son muy resistentes a la sequía y crecen en áreas climáticas secas (Galera, 2000).

De acuerdo con las investigaciones de Villagra et al. (2010), en las zonas áridas con precipitaciones se desarrollan en distintos tipos de suelos, especialmente en el franco arenoso. Crecen mejor en arenas permeables, pero a veces se los encuentra en arcillas, arenas arcillosas y aun en suelos salinos, habitando en serranías sobre suelos con cierta pedregosidad o aluvionales hasta los $1000 \mathrm{~m} \mathrm{snm}$ (Galera, 2000).

Según Venier et al. (2013) los cambios en las condiciones climáticas que se registrarían hacia el año 2050 afectarían marcadamente la distribución de los bosques de los morfotipos de $P$. alba. Como patrón general se observa que en un escenario futuro se producirá una migración hacia el sur de los morfotipos "Chaqueño norte" y "Santiagueño", mientras que el morfotipo "Chaqueño sur" se desplazaría hacia el norte. El escenario planteado supone un aumento promedio de las temperaturas mínima y máxima de $1^{\circ} \mathrm{C}$, pero también un aumento promedio en las precipitaciones anuales de $55 \mathrm{~mm}$. Dado que esta especie está especialmente adaptada a climas áridos, esto podría estar indicando que el efecto del cambio climático sobre la especie podría estar más influido por un aumento en las precipitaciones que por un aumento de la temperatura. En cuanto a estudios realizados para los morfotipos "Chaqueño norte" y "Chaqueño sur", se observa que sus respectivos rangos de distribución se verían considerablemente afectados. Aunque surgirían áreas favorables de una extensión igual o mayor a la actual, en particular $P$. alba "Chaqueño sur" se desplazaría hacia el norte, en contraposición a la tendencia general observada en los otros morfotipos. Este morfotipo se estaría desplazando no por un incremento de las precipitaciones (como ocurriría en los otros casos), sino por el contrario, por un incremento en las condiciones de estrés hídrico (Venier et al., 2013).

Por su parte, el morfotipo "Santiagueño", sería el menos afectado bajo el escenario de cambio climático; ya que se observa que la mayor parte de su distribución actual permanecería estable, sin sufrir retracciones ni fragmentaciones. Esto podría deberse a que es el morfotipo que estaría más adaptado a una alta variabilidad de temperatura según su actual área de distribución en sentido norte-sur coincidente con el sentido en que varían las temperaturas en la región de estudio. Además, sería significativamente más tolerante al estrés hídrico (López-Lauenstein et al., 2010), lo que podría explicar en parte este resultado.

Como representante de la familia Fabaceae, $P$. alba puede establecer relaciones simbióticas con diferentes géneros de rizobacterias (Di Marco, 2013) formando nódulos de tipo indeterminado, en los cuales ocurre la fijación biológica del nitrógeno atmosférico $\left(\mathrm{N}_{2}\right)$. Estas relaciones en general actúan sobre las respuestas de tolerancia y escape a la sequía, mejorando el rendimiento del fotosistema II y disminuyendo la pérdida de hojas; mejora las condiciones fisiológicas en general, incrementando los niveles de antioxidantes no enzimáticos, prolina y clorofilas (Pozzi-Tay, 2016).

\section{BIENES NO MADEREROS Y SERVICIOS PROPORCIONADOS POR EL ALGARROBO}

Antes de la llegada de los europeos, los algarrobos arbóreos como $P$. alba, constituían una fuente importante de alimentos, combustible y materiales de construcción para los hogares. También constituyó un 
valioso recurso para los primeros colonos europeos que consumieron las vainas con alto contenido de azúcar durante las sequías y que usaron la madera para carbón y materiales de construcción (D'Antoni \& Solbrig, 1977). Actualmente, y acorde con los productos y servicios que genera, se la define como una especie multipropósito. Demaio et al. (2015) la describen como una especie forestal de gran importancia regional debido a la diversidad de usos tanto madereros como alimenticios, forrajeros y medicinales.

El algarrobo blanco podría constituir una importante alternativa productiva en la economía de la familia campesina del norte de nuestro país, proporcionándole materias primas para diversos fines.

Servicios ecosistémicos, mejoradora de ambientes Desde tiempos históricos el hombre utilizó el Prosopis alba en diversos usos cotidianos (Fagg \& Stewart, 1994; Villagra \& Morales, 2003) siendo una especie multipropósito (Giménez et al., 2001; López, 2005; Di Marco, 2013). Esta especie brinda servicios indirectos como: moderación de las temperaturas extremas, disminución de la evapotranspiración, amortiguamiento y redistribución en la caída de las precipitaciones, incremento de la fertilidad de suelos por acumulación de nutrientes; mejora de la estructura del suelo por efecto del mantillo; provisión de "perchas" para aves dispersoras de frutos de otras especies (Villagra, 2000). En función de sus características de crecimiento, el algarrobo blanco tiene potencial para ser usado en la restauración de áreas degradadas y puede catalizar el desarrollo de nuevos sistemas de producción en los ecosistemas áridos argentinos (López, 2005; Taleisnik \& López-Lauenstein, 2011). En sistemas productivos, su copa ofrece sombra para el ganado. Ocampo (2011) sostiene que la disminución de la temperatura bajo el dosel de copas reduce la carga calórica de los animales, con lo que se logran beneficios en la conducta, la reproducción y la sobrevivencia de los animales, y en definitiva aumenta la productividad. Por otro lado, su cultivo es conveniente desde el punto de vista medioambiental ya que, por ser una leguminosa, fija nitrógeno en el suelo, aportando entre 100 a 400 $\mathrm{kg} / \mathrm{ha} / \mathrm{año}$, y resulta ser una especie mejoradora de suelos salinos (Sciammaro, 2015).

\section{Alimentación humana}

Los frutos de los Prosopis spp. son considerados como importantes recursos alimenticios en regiones áridas y semiáridas del mundo, con un contenido de proteínas entre 11 y $17 \mathrm{~g} / 100 \mathrm{~g}$ MS teniendo como aminoácidos limitantes tirosina y metionina/cistina y de 13 a $34 \mathrm{~g} / 100 \mathrm{~g}$ MS de carbohidratos, siendo el azúcar principal la sacarosa (Meyer et al., 1986).

En Argentina, la utilización de los frutos de algarrobo (algarroba) en la alimentación humana se remonta a las culturas prehispánicas. En la Provincia de Santiago del Estero los pobladores locales preparaban alimentos tradicionales como "patay", "aloja" y "añapa" (Ochoa de Cornelli, 1996). En la Provincia de Mendoza, los indios Huarpes, al no disponer de trigo, maíz o arroz para elaborar el pan, utilizaban algarroba que cosechaban y almacenaban (Rusconi, 1962). En la Provincia de Chaco, los Tobas preparaban harina con las algarrobas de $P$. alba y $P$. nigra, dejándolas secar y moliéndolas en un mortero para consumirlas disuelta en agua (MartínezCrovetto, 1964). Su uso en alimentos humanos responde a su sabor agradable y su alto valor nutritivo.

Existe información sobre la composición química de frutos (Galera et al., 1992; Prokopiuk et al., 2000), pulpas obtenidas a partir del secado y molienda de las vainas sin semillas (Rozycki et al., 1998), y semillas (Lamarque et al., 1994) de $P$. alba.

Prokopiuk et al. (2000) reportan la composición de frutos enteros (con semillas) de $P$. alba molidos y secados a 60 ○C por 60 minutos. Expresados en gramos por kilogramo de masa seca contienen proteínas: 71,7 , grasa: 21,7 , cenizas: 31,3 , carbohidratos totales: 849,6 , azúcares totales: 591,4 , azúcares reductores: 27,6 , fibra cruda: 24,3, fibra dietética insoluble: 200,9, fibra dietética soluble: 64,7 , fibra dietética total: 265,6, taninos condensables: 5,7, polifenoles solubles totales: 0,06, Calcio: 1,2745, Hierro: 0,450, Potasio: 8,920, y Magnesio: 0,967.

Entre los factores antinutricionales (tripsina, ácido fítico, taninos), los taninos son comunes en las semillas de leguminosas y se han hallado en cantidades considerables en algunas especies del género Prosopis. Dada la baja proporción de taninos en frutos de $P$. alba, son insignificantes los efectos adversos de los taninos condensados $(5,7 \mathrm{~g} / \mathrm{kg}$ base seca) y polifenoles solubles totales $(0,06 \mathrm{~g} / \mathrm{kg}$ base seca). Por lo tanto, su presencia no constituye un factor limitante para ser usado en la elaboración de alimentos (Prokopiuk et al., 2000); aunque se han informado efectos tales como disminución de digestibilidad de proteínas, depresión de crecimiento e inhibición de enzimas digestivas (Singleton, 1981).

González-Galán et al. (2008) detectaron valores bajos de actividad hemaglutinante en $P$. alba. El simple hecho de presentar actividad hemaglutinante baja no nos permite descartar la posibilidad de estar frente a una lectina tóxica. Las lectinas disminuyen la absorción de nutrientes, provocan pérdida de proteína, rápida pérdida de peso e inhibición de crecimiento; además, muestran capacidad de inhibir varias enzimas intestinales.

Uno de los productos comerciales derivado de los frutos es la harina de algarroba (HA). El Código Alimentario Argentino define a la HA como el producto de la molienda de las semillas, limpias, sanas y secas, del algarrobo blanco (Prosopis alba) y/o algarrobo negro (Prosopis nigra). Asimismo, se entiende como harina de fruto (vaina completa con sus semillas) de algarrobo, al producto de la molienda de los frutos completos limpios, sanos y secos, del algarrobo blanco y/o algarrobo negro. La HA tiene una menor cantidad de proteína que la harina de trigo, mientras que es mayor la cantidad de grasa, cenizas y fibra. El contenido de fibra es casi 10 veces mayor $y$, por consiguiente, es menor el contenido de carbohidratos disponibles, lo que resulta en una importante disminución del valor calórico, casi un $50 \%$ menor que el de la harina de trigo. La composición de la HA estudiada se asemeja a la de otras especies de Prosopis, caracterizadas por importantes aportes de fibra dietaria y niveles moderados de proteínas si bien los contenidos absolutos pueden variar, reflejando la biodiversidad y la singularidad de cada especie (Zuleta et al., 2012).

Las buenas propiedades fisicoquímicas y nutricionales de la HA hacen que la misma constituya un ingrediente 
ideal para la elaboración de diferentes productos horneados dulces saludables (Sciammaro, 2015). Para la elaboración de galletas, es tecnológicamente posible sustituir hasta un $20 \%$ de harina de trigo por HA mejorando la calidad proteica, incrementando el aporte de fibra y minerales de los productos obtenidos con las mezclas (Macías et al., 2013). Otros estudios efectuados en Argentina también muestran la factibilidad de reemplazar la harina de trigo por HA de Prosopis alba, hasta el $4 \%$ en la obtención de pan francés y pan de molde, y hasta el $12 \%$ en el caso de galletitas dulces (Rozycki et al., 1998).

La HA presenta buena solubilidad y absorción en agua, posee sólidos solubles para harinas integrales cruda y tostada de 65,00 y $33,25 \mathrm{~g} / 100 \mathrm{~g}$ respectivamente (Margalef et al., 2012).

Se utilizan también las algarrobas para la elaboración del "patay" o pasta harinosa obtenida al moler la algarroba madura y seca en mortero y pasarla por cedazo fino. Es un producto dulce (17,2 \% glucosa y $26,9 \%$ sacarosa) elaborado con harina fina y seca que se mezcla con agua hasta formar una masa. Esta masa se introduce en moldes circulares y se hornea. Se vende en mercados regionales y constituye un importante alimento regional en Santiago del Estero y otras provincias de Argentina (Galera, 2000).

De las algarrobas puede obtenerse un sustituto del café. En Perú, a partir de la molienda de frutos de especies nativas del género, Grados \& Cruz (1996) han analizado las diferentes fracciones obtenidas para la elaboración de variados subproductos, como sustituto del café. Prokopiuk (2004) ha ensayado procesos controlados de molienda para la obtención de sustitutos de café a partir de frutos de $P$. alba. Al respecto, Hieronymus (1882) ya cita que las semillas tostadas podrían reemplazar al café. Actualmente, entre los productos derivados existentes en el mercado, figura el sustituto del café (granos o polvo soluble instantáneo) como una alternativa saludable (Mom, 2012). El aroma de la infusión resultante presenta matices de tostado, miel, hierba y chocolate. Resulta parecido al del café genuino y tuvo aceptación entre los consumidores. Recientemente, algunas pequeñas fábricas en Perú comenzaron a elaborar sucedáneos de café o café de algarroba (Cruz, 1999). En la Provincia de Formosa (Argentina), este producto es fabricado y comercializado por pobladores originarios de la etnia Wichi. El café de algarroba es un producto derivado de las fracciones 3 y 4 de la molienda de la chaucha de algarrobo (Traskauskas et al., 2001).

También se puede producir alcohol etílico de buena calidad a partir de la fermentación de los frutos de algarrobo, con un rendimiento de 27 litros de alcohol absoluto por cada $100 \mathrm{~kg}$. Es factible la elaboración de una bebida alcohólica, la "aloja" (Galera, 2000).

La aloja es una bebida fermentada típica de la región del noroeste argentino elaborada con vainas enteras de algarrobo blanco. Las vainas de Prosopis alba se muelen en un mortero y se introducen con agua en una vasija para favorecer el proceso de fermentación. La mezcla se guarda en lugar fresco, seco y en oscuridad. Luego de dos días, los pedazos de vainas se remueven en forma manual, se descartan, y se agregan nuevas vainas de modo de favorecer la fermentación. El punto óptimo de la bebida se detecta por cata o degustación y su período de preservación es muy corto. Otra bebida tradicional, pero analcohólica, es la añapa; su elaboración se inicia de forma similar a la aloja (mezclando vainas molidas en mortero con agua) pero se evita su fermentación logrando una bebida fresca y dulce. Generalmente se consume por sus propiedades diuréticas (Sciammaro, 2015).

Otro preparado artesanal y comercial es el arrope, algarrobina o miel de algarrobo. Es un líquido denso y oscuro obtenido luego de machacar las vainas de algarroba con agua y cocinar la mezcla durante 8 horas. Durante este proceso se produce la concentración de azúcares. En la mitad de la cocción se separan las semillas. Se obtiene un jarabe oscuro tipo miel (Sciammaro, 2015).

También tiene valor comercial la goma de algarroba. Su extracción puede ser por los métodos químico alcalino o mecánico (en seco). Se trata de un hidrocoloide con propiedades funcionales que lo convierten en un aditivo con capacidad espesante y estabilizante, adecuado para la incorporación en sopa de arvejas y postres sabor a dulce de leche, con características sensoriales aceptables y sin sinéresis (Millán et al., 2016).

Como producto de desecho en el proceso de obtención de harinas de vainas se comenzaron a estudiar las semillas de $P$. alba encontrando que las mismas tienen propiedades nutricionales y funcionales. Entre los fitoquímicos extraídos se destacan fenólicos solubles totales 5,05-8,58 mg EAG/g harina, fenólicos flavonoides precipitados $0,10-0,31 \mathrm{mg} \mathrm{EQ} / \mathrm{g}$ harina, no flavonoides 2,68-4,01 mg EAG/g harina, observándose mayor variación en el contenido de taninos condensados 44,75$223,72 \mathrm{mg} \mathrm{EPB} / \mathrm{g}$ harina, metabolitos con propiedades antioxidantes, pero a la vez considerados como antinutricionales. Se identificaron 3 flavonoides Cglicósidos y un ácido fenólico. Presentaron actividad antioxidante permitiendo identificar propiedades beneficiosas para la salud humana (Correa-Uriburu et al., 2017).

\section{Uso medicinal}

Entre las aplicaciones medicinales de Prosopis alba pueden mencionarse que los frutos tienen la propiedad de disolver los cálculos de vejiga. También se emplean como antibronquial y laxante. Emplastos de frutos verdes, después de separar las semillas, curan fracturas de huesos sin herida (Hieronymus, 1882). En el centro y norte de la Provincia de Santa Fe su savia se utiliza para combatir "el mal de ojo".

Sus hojas procesadas se utilizan para curar las rescaldaduras y quebraduras de los huesos; el macerado de las mismas se usa como antiséptico (Amsler, 1986). Sus flores tienen propiedades diuréticas, y la corteza se utiliza como antidiarreico y contra las oftalmías (Giménez et al., 2016).

\section{Alimentación animal}

En Argentina, el uso de diferentes especies de Prosopis para alimento de animales se remonta a siglos atrás (Galera, 2000). Di Marco (2013) menciona que las hojas y frutos de algarrobo blanco pueden utilizarse como forraje. Sus hojas registran un $22 \%$ de proteína bruta, $15 \%$ de proteína digestible y un $55 \%$ de digestibilidad de la materia seca (Galera, 2000). Coe \& Coe (1987) hacen referencia a la alta palatabilidad de los frutos, lo cual sumado a su calidad nutricional: 25 a $28 \%$ de 
glucosa, 11 a $17 \%$ de almidón, 7 a $11 \%$ de proteínas, bajo tenor graso y buena digestibilidad, convierten al recurso en un excelente forraje (Fagg \& Stewart, 1994). Los ensayos sobre el valor nutritivo de los frutos de algarrobo blanco indican que se trata de un alimento adecuado y comparable al de otras especies, como el maíz, para ser utilizado como suplemento (Brunetti et al., 2001). Puede usarse tanto en pastoreo directo $y / 0$ diferido para suplemento en invierno. Galera (2000) menciona su uso para ganado vacuno, caballar, caprino y porcino; citando a Briones (1985), señala a la especie como una fuente fundamental de nutrición tanto para animales salvajes como domésticos. Díaz (1962) refiere a su utilización en Tucumán, Argentina, para la alimentación del ganado lechero. En trabajos realizados en distintos puntos de nuestro país se observó que con ramoneo de Prosopis alba se han registrado mejoras en el aporte de proteína bruta a la dieta de vaquillonas en sistemas silvopastoriles en bosque nativo (Saravia Sánchez, 2012).

Tagliamonte et al. (2014) recomienda la administración de frutos molidos, para aprovechar los contenidos en proteína y grasa de las semillas. En este sentido, a pesar de que la harina de la vaina del Prosopis sea ampliamente utilizada en la elaboración de bebidas, dulces y substitutos del café (Meyer et al., 1986; Bravo et al., 1998; Bernardi et al., 2006) los trabajos relacionados al estudio de los antinutrientes en ella son muy escasos. Particularmente en conejos, se recomienda utilizar hasta el $14 \%$ de harina en la alimentación durante las etapas de crecimiento-engorde, ya que debido a su alto contenido de fibra cruda se vuelve más lenta la digestión determinando un menor consumo de alimento (Macías-Rodríguez \& UscaMéndez, 2017). La importancia del uso de $P$. alba para la alimentación animal se evidencia al considerar que recientemente (2015) se inició una linea de mejoramiento genético en la especie para la producción de vainas como forraje (López-Lauenstein et al., 2015). Además, Macías-Rodríguez y Usca-Méndez (2017) sugieren aprovechar al máximo la producción de esta leguminosa, con la finalidad de que sirva para la alimentación de otras especies pecuarias.

\section{Uso industrial}

Tortorelli (2009) cita que los primeros brotes foliares podrían utilizarse en el perfume de cueros debido a los aceites volátiles e hidrocarburos que le confieren un interesante aroma.

Asimismo, su resina es conocida como excelente tintura de color negro para lana o algodón. En 2015 se logró la extracción de sustancias colorantes de $P$. alba resultando aptas para el teñido de textiles con métodos simples y buena solidez del color. Esta ha sido una iniciativa de INTI Formosa e INTI Textiles con el objetivo de capacitar a las comunidades Wichi y Toba en la aplicación de estos colorantes (Jarzinski et al., 2015).

Se trata de un pigmento para fibras proteicas y celulósicas de uso en industria textil y posiblemente como colorante alimenticio; obtenido a partir de extracto de chauchas de Prosopis. El producto es un polvo de color violeta-rojizo que puede utilizarse como ingrediente. Es soluble en agua. Se ha testeado que puede ser envasado en envases herméticos de vidrio o films impermeables (Álvarez et al., 2015).

\section{RECURSO MADERABLE}

Prosopis alba es una de las especies nativas de mayor uso para madera de aserrío en Argentina (Venier et al., 2013). Según la Secretaria de Ambiente y Desarrollo Sustentable de la Nación, en 2009 se extrajeron 160 mil toneladas; ordenados según su importancia, los productos fueron rollizos, leña, rodrigones, postes y trocillos. Di Marco (2013) menciona que, de dicha cantidad, más del $70 \%$ provinieron de la región chaqueña: para Chaco se estima una producción de 100 mil toneladas anuales con destino a la producción de muebles. En 2010, las estadísticas de dicha provincia contabilizan 51.491 toneladas de rollos según el cómputo de guías y vales de transporte (Secretaría de Planificación y Evaluación de Resultados de la Provincia del Chaco, 2010). Asímismo, la infografía generada por el Ministerio de Ambiente y Desarrollo Sustentable de la Nación Argentina (2020) coloca al algarrobo como una de las especies más utilizadas para la producción de rollizos en el período 2007-2016 en el Parque Chaqueño. Dado que la totalidad de la madera de algarrobo consumida proviene de bosques naturales, la explotación del recurso resulta insostenible, no sólo desde el punto de vista ambiental sino también económico (Venier et al., 2013). Si bien en la región chaqueña la plantacion del algarrobo ha sido iniciada hace más de veinte años, la ausencia de una base cientifico-tecnológica de la actividad y las dificultades de buena parte de las plantaciones debidas a la falta de materiales adecuados de propagacion, la escasa experiencia de manejo y seguimiento, han determinado que el reemplazo de la madera de aserrío proveniente del bosque nativo por madera de bosques cultivados sea parcial. En este contexto, el Programa Forestal del INTA, a partir de 2006, se propuso generar información y material de propagación de $P$. alba orientando su accionar a la búsqueda de un mayor potencial de crecimiento para la producción de madera de calidad destinada al aserrío (López-Lauenstein et al., 2015).

La especie en cuestión, junto al algarrobo negro (Prosopis nigra), han sido excesivamente explotadas en toda su área de distribución. Esta explotación se ha caracterizado por la extracción de los mejores ejemplares arbóreos, limitando drásticamente la capacidad para generar productos leñosos y alimenticios en la cantidad y la calidad que demanda la población. Por otra parte, los sistemas de producción agrícola de Argentina aspiran a obtener altas producciones y de alto valor exportable, modelos que no sólo se llevan a cabo en ambientes productivos como la región Pampeana, sino que se expanden hacia ambientes marginales como el Espinal y el Chaco. Para lograrlo deben modificar los ambientes adaptándolos a los modernos sistemas de producción y eliminando los estratos de vegetación, uniformando de esta manera el espacio, por lo que los bosques ceden paso a praderas agrícolas o ganaderas (Karlin, 1988; López, 2005). Estas actividades trajeron aparejada la pérdida de germoplasma que compromete el potencial evolutivo de las especies y limita la capacidad de recomponer su productividad (Salto, 2011).

Las características tecnológicas de la madera de algarrobo blanco permiten su uso para muebles, tonelería, aberturas, parquet, carpintería rural, leña y 
carbón (Felker, 1999). El uso extendido en la industria del mueble se debe a las excelentes propiedades físicomecánicas de su madera: muy estable, densa y de baja contracción volumétrica (Araujo et al., 2003). Es una especie de gran potencial para su utilización como componente forestal en sistemas silvopastoriles; de gran potencial ya que se adapta perfectamente al permitir que las pasturas prosperen bajo su dosel. La amplia copa no densa aporta materia orgánica y nutrientes, en especial nitrógeno. El sistema radical no es competitivo con gramíneas (Karlin et al., 1997).

\section{Producto maderero}

El Anuario de Estadística Forestal 2017-2018 lista los productos forestales primarios por especie extraidos en el mencionado período. De forma global y consignando únicamente como productos de "algarrobo", menciona que se extrajeron $93.368 \mathrm{~m}^{3}$ de rollizos, $89.636 \mathrm{tn}$ de rollos, 106.640 tn de leña como tal y 160 tn de leña para carbón; la extracción discriminada por especie $(P$. alba) se presenta sólo para algunos productos: $249 \mathrm{~m}^{3}$ de rollizos y 239 tn de rollos (Secretaría de Gobierno de Ambiente y Desarrollo Sustentable, 2019).

La corteza es marrón-rojiza y, luego de cortada, se torna marrón oscura. La albura es de color blanco amarillento y el duramen castaño rosado. Presenta gran cantidad de parénquima longitudinal y radios anchos; su textura varía de mediana a gruesa (Galera, 2000). Su veteado es pronunciado, floreado suave en el corte tangencial y jaspeado tenue en el corte radial. Es una madera moderadamente pesada a pesada, con una densidad aparente al $15 \%$ de humedad de $0,760 \mathrm{~kg} / \mathrm{dm}^{3}$. Resistente al ataque de hongos e insectos, se clasifica como madera durable. Una de sus mejores cualidades es la gran estabilidad dimensional. Muestra buen comportamiento durante el secado, sin deformaciones, tanto en el proceso natural como artificial. No ofrece dificultades para el aserrado en verde y permite superficies lisas y pulidas. Debido a su dureza presenta dificultades para el clavado. Requiere pretaladrado. Fácilmente penetrable con regular absorción de líquidos impregnables. Toma bien tintes, lustres y barnices (Di Marco, 2013). De color más oscuro que otros algarrobos, dura para clavar y permeable a tratamientos de impregnación. Posee buena respuesta al cepillado y posibilidades de debobinar (Galera, 2000).

FAO (1985) menciona que la duración de la madera es independiente de la edad; la permeabilidad de la albura es mayor que la del duramen y tiende a incrementarse con la edad. La capacidad mecánica puede incrementarse con la edad del árbol, en cambio, no es significtivo el incremento de la densidad de la madera. Por su parte, Giménez et al. (1998) realizaron un análisis epidométrico y consideraciones anatómicas en $P$. alba; estos estudios permitieron determinar que los anillos de crecimiento tienen un espesor medio de $4,05 \mathrm{~mm}$, que el espesor medio de la albura es de 3 (2-6) anillos, no existiendo correlación entre la albura y la edad; que el espesor medio de corteza es 1,6 mm $(0,39 \mathrm{~mm}$ corteza viva y $0,74 \mathrm{~mm}$ corteza muerta) y el proceso de duraminización se inicia entre los 3-6 años. La proyección del incremento medio anual (IMA) en el volumen se interceptaría a una edad de 25 años, pudiendo interpretarse como el turno tecnológico de corta.
En Argentina más del $60 \%$ de los muebles de algarrobo son elaborados con madera de Prosopis alba (Gimenez et al., 1998). Se la emplea en la elaboración de muebles de madera maciza de excelente calidad, fabricación de marcos de puertas y ventanas. También se usa para la elaboración de barriles de vino, hormas de zapatos y parquets (Tortorelli, 2009), y productos como platos, utensilios, cajas, adornos, etc., son realizados por artesanos locales con algarrobo (Galera, 2000).

Como combustible es un recurso excelente debido a su poder calorífico elevado, de combustión lenta y escasa ceniza (Di Marco, 2013). Su leña produce $4200 \mathrm{kcal} / \mathrm{kg}$, y el poder calorífico de su carbón es de $6500 \mathrm{kcal} / \mathrm{kg}$, con una eficiencia de transformación donde 4-5 tn de leña equivalen a 1 tn de carbón (Galera, 2000). Aparte de estas aplicaciones, la madera de duramen puede proporcionar del 10 al $12 \%$ de su peso de un extracto tánico de buena calidad, especial para curtir pieles finas a las que otorga consistencia suave y flexible (Zurloaga et al., 2008; Tortorelli, 2009).

\section{Situación tecno-productiva de la madera de $\boldsymbol{P}$. alba}

La principal materia prima que abastece a los eslabones productivos proviene fundamentalmente del bosque nativo de la Provincia del Chaco y en algunos casos del interior de Corrientes y Formosa, donde se destaca el algarrobo como madera más utilizada (Bonfanti, 2014). La producción del sector se orienta principalmente a productos artesanales y mobiliario rústico en base a maderas nativas (Federico, 2016). Se aprecia que la ventaja inicial de contar con abundancia de materia prima, básicamente algarrobo, hoy no es tal, sino que se está próximo a la escasez del recurso. Las estimaciones más conservadoras hablan de un horizonte de abastecimiento que alcanza sólo para los próximos 10 años. La evidencia de ello es el alza exponencial de su precio. En consecuencia, la necesidad de trabajar con madera alternativa reducirá considerablemente la ventaja comparativa del bajo requerimiento de capital de trabajo en virtud de que ésta, a diferencia del algarrobo, requiere secado.

El creciente desmonte, que tiene como fin en muchos casos el uso de la tierra para la producción de cultivos anuales, ha hecho que hoy el algarrobo se esté acabando (incluso en áreas donde su explotación está prohibida, como a orillas de los ríos). Ello obliga a las empresas de aserrado a pagar más o bien a migrar hacia otros tipos de materiales. En ambos casos se ve afectada en forma directa tanto la rentabilidad empresarial como la sustentabilidad del recurso. La migración al uso de otras especies requiere indudablemente incorporar un proceso adicional: el secado. Para ello se debe stockear, lo que implica un costo financiero adicional (Secretaría de Política Económica - Programa de Competitividad del Norte Grande, 2009).

El informe referido a las características de la región Parque Chaqueño (Ministerio de Agroindustria, s/fecha) menciona que en ella la principal especie promocionada para la forestación es el algarrobo. En la Provincia de Formosa se han inspeccionado 1.174 ha forestadas con algarrobo (2000-2018), tanto en macizo como en enriquecimiento (en la zona noreste de la Provincia predominan los macizos mientras que en el oeste el 
enriquecimiento). En la Provincia de Chaco se ha implementado un programa de promoción provincial (Plan Provincial de Expansión Forestal 1991 - 2005), con el cual se ha financiado la plantación de 2.930 ha de algarrobo. A partir del año 2000 y hasta la fecha, la promoción forestal se realiza a través de la ley 25.080 (1998) y sus modificatorias las leyes 26.432 (2008) y 27.487 (2018), inspeccionándose 589 ha de forestación con algarrobo. Los datos más actuales son los proporcionados por Salto y Lupi (2019) quienes estiman que existen alrededor de 9.000 ha forestadas con algarrobo blanco, sin considerar aquellas forestaciones que se establecieron fuera del marco de la Ley 25.080. Con relación a las especies forestales nativas recomendadas para la Provincia, el desarrollo de un paquete tecnológico elaborado hasta la actualidad está dirigido principalmente a Prosopis alba (algarrobo blanco) (Bercovich, 2000).

La industria y la comercialización en la región Chaqueña se llevan a cabo con madera del bosque natural. Existen dos tipos de empresas: por un lado, los pequeños aserraderos y carpinterías, sin inversión en infraestructura, marketing o comercialización, que venden localmente; $y$, por otro lado, los grandes aserraderos, que cuentan con integración horizontal y vertical, los cuales venden dentro del país e incluso al exterior (principalmente Italia, Estados Unidos y España) (Bercovich, 2000). Por otra parte, algunas de las forestaciones de algarrobo implantadas en la zona han tenido cortas intermedias y sus productos se han utilizado en el mismo predio/territorio en forma de postes de diversas dimensiones o como leña o materia prima para la elaboración de carbón; además los diámetros mayores han sido comercializados en aserraderos y carpinterías (Víctor Pérez, com. pers., 2018).

\section{CONCLUSIONES}

Prosopis alba es una de las especies nativas más importantes de nuestro país y tiene una extensa área de distribución que incluye diversos ecosistemas forestales nativos que se extienden a países limítrofes; debido a su abundancia y comportamiento ecológico, es de gran importancia ecosistémica en zonas áridas y semiáridas del mundo y de vital importancia en su economía rural. Es considerada una especie de uso múltiple por la utilidad de su madera, frutos, propiedades medicinales, entre otros servicios directos e indirectos que brindan a la sociedad. En función de sus características de crecimiento y adaptación, ha sido valorado su potencial para ser empleada en la restauración de áreas degradadas por salinización inclusive, y puede catalizar el desarrollo de nuevos sistemas de producción en los ecosistemas áridos argentinos; ya sea con el establecimiento de plantaciones puras como en sistemas integrados como los silvopastoriles.

Comercialmente es muy valorada por su madera, debido a las excelentes propiedades físico-mecánicas: muy estable, densa y de baja contracción volumétrica.

Si bien reviste importancia económica y social en la industria forestal y como creadora de ingresos para la región de producción, aún es necesario generar información relacionada al potencial productivo de los diferentes sitios en los que actualmente se desarrollan las plantaciones con esta especie; además queda evidenciada la dificultad para acceder a información actualizada relacionada a superficie implantada del recurso y la situación técnico-productiva de su madera.

\section{BIBLIOGRAFÍA}

Álvarez H., D. Vergara, M. Jarzinski, R. Velazco \& R. Baeza. 2015. Obtención de colorantes naturales a partir de semilla de algarroba para uso en fibras textiles y alimentos. En: INTI (Ed.), TecnoINTI: $12^{\circ}$ Jornadas Abiertas de Desarrollo, Innovación y Transferencia Tecnológica. Instituto Nacional de Tecnología Industrial, Buenos Aires. pp.158.

Amsler, A. 1986. Árboles, Arbustos y Hierbas de la Provincia de Santa Fe, su Aplicación Medicinal. Instituto de Investigaciones de Productos Naturales de Análisis y Síntesis Orgánica. Ministerio de Agricultura y Ganadería de la Provincia de Santa Fe, Santa Fe, Argentina. 102 pp.

Araujo, P.A., A. Remacha-Gete, J.C. Medina \& J.R. Taboada. 2003. Los recursos maderables del Chaco Semiárido Argentino. Características, usos actuales y potenciales. Revista AITIM 224: 50-53.

Barros, S. 2010. El género Prosopis, valioso recurso forestal de las zonas áridas y semiáridas de América, Asia y África. Ciencia e Investigación forestal 16(1): 91127.

Bercovich, N. 2000. Evolución y situación actual del complejo Forestal en Argentina. División de desarrollo productivo y empresarial de la Comisión Económica para América Latina y el Caribe. Centro Internacional de Investigaciones para el desarrollo (CIID/IDRC). Argentina. $65 \mathrm{pp}$.

Bernardi, C., S. Drago, N. Sabbag, H. Sanchez \& M. Freyre. 2006. Formulation and sensory evaluation of Prosopis alba (algarrobo) pulp cookies with increased iron and calcium dialyzabilities. Plant Foods for Human Nutrition 61(1): 37-42.

Bessega, C., B.O. Saidman \& J.C. Vilardi. 2005. Genetic relationships among American species of Prosopis (Leguminosae) based on enzyme markers. Genetics and Molecular Biology 28(2): 277-286.

Bonfanti, F. 2014. Las políticas de desarrollo local dentro del complejo económico de la madera en el Chaco. El caso del programa Veta Noble. Disponible en http://hum.unne.edu.ar/revistas/geoweb/Geo26/archivos /congreso\%20geografia/Exposiciones/Exposiciones\%20 Eje\%202/Bonfanti_EJE2.pdf. Último acceso: mayo 2019. Bravo, L., N. Grados \& F. Saura-Calixto. 1998. Characterization of syrups and dietary fiber obtained from mesquite pods (Prosopis pallida L). Journal of Agricultural and Food and Chemistry 46: 1727-1733.

Briones, L. 1985. Visión Retrospectiva Antropológica del Prosopis. En: M. Habit (Ed.), Estado actual sobre el conocimiento de Prosopis tamarugo. Arica, Chile. pp. 1115.

Brunetti, M., M.P. Silva, R. Coirini \& M.J. Martínez. 2001. Valor nutritivo del fruto del algarrobo blanco (Prosopis chilensis) como suplemento forrajero para ser utilizado en cabras. Revista Argentina de Producción Animal 21(1): 23-24. 
Burkart, A. 1976. A monograph of the Genus Prosopis (Leguminosae Subfam. Mimosoideae). Journal Arnold Arboretum 57(3-4): 219-525.

Carnevale, J. 1955. Árboles forestales. Descripción, cultivo, utilización. Ed. Hachette AS. Argentina. 689 pp.

Coe, C. \& M. Coe. 1987. Large herbivores, acacia trees and bruchid beetles. South African Journal of Science 83(10): 624.

Correa-Uriburu, F.M., M.R. Alberto \& M.I. Isla. 2017. Estudio fitoquímico y actividad biológica de semillas de diferentes clones de Prosopis alba. Dominguezia 33(1): 82.

Cruz, G. 1999. Production and characterization of Prosopis seed galactomannan. Tesis Doctoral. Swiss Federal Institute of Technology, Zurich. $118 \mathrm{pp}$.

D'Antoni, H.L.D. \& O.T. Solbrig. 1977. Algarrobos in South American cultures: past and present. En: B.B. Simpson (Ed.), Mesquite. Its biology in two Desert Scrub Ecosystems. Dowden, Hutchinson and Ross, Stroudsburg, Pennsylvania, USA. pp.1-26.

Delvalle, P., M. Atanasio, M. Ayala, I. Svriz \& J. Petkoff. 2003. Ensayo de orígenes de Prosopis alba Griseb. (Algarrobo blanco). Disponible en https://inta.gob.ar/sites/default/files/script-tmp-intaensayo_de_orgenes_de_prosopis_alba_griseb_alga. pdf. Último acceso: mayo 2019.

Demaio, P., U. Karlin \& M. Medina, M. 2015. Árboles nativos de Argentina (Vol. 1). Ecoval Ediciones. Córdoba, Argentina. 188 pp.

Díaz, H.B. 1962. Especies arbóreas más comunes de las zonas ganaderas de la Provincia de Tucumán que sirven de alimento al ganado. Turrialba 12(4): 195-199.

Di Marco, E. 2013. Prosopis alba Griseb. (Algarrobo Blanco) (Familia Fabaceae, Mimosoideas). Ficha Técnica. Área Técnica Promoción Dirección de Producción Forestal MAGyP. Disponible en http://forestoindustria.magyp.gob.ar/archivos/procedimie nto-requerido-en-plantaciones/prosopis-alba-grisebfamilia-fabaceae-mimosoide.pdf. Último acceso mayo 2019.

Fagg, C.W. \& J.L. Stewart. 1994. The Value of Acacias and Prosopis in Arid and Semi-arid environment. Journal of Arid Environments 27: 3-25.

FAO. 1985. Durabilidad natural y permeabilidad de la madera de tamarugo (Prosopis tamarugo Phil.) y algarrobo (Prosopis alba Griseb.). Panel 4: tecnología (continuo). En: Mesa Redonda Internacional sobre Prosopis tamarugo Phil. Arica, Chile, 11-15 Junio 1984.

Federico, J. 2016. Análisis tecnológicos y prospectivos sectoriales. Maderas y muebles. Ministerio de Ciencia, Tecnología e Innovación Productiva. Buenos Aires, Argentina. $63 \mathrm{pp}$

Felker, P. 1999. Oportunidades de Inversiones en el Algarrobo (Prosopis alba). Secretaría de Producción y Medio Ambiente. Santiago del Estero, Argentina. 13 pp.

Galera, F.M. 1995. Banco de Germoplasma para especies del género Prosopis de la Provincia de Córdoba - Argentina: Organización y Estado Actual. En: Memorias XIV R.G.C FAO - INTA. pp.54-63.

Galera, F.M. 2000. Las especies del género Prosopis (algarrobos) de América Latina con especial énfasis en aquellas de interés económico. UNC-Secretaría de Ciencia y Tecnología. Córdoba, Argentina. 269 pp.

Galera, F.M., M. Trevisson \& S. Bruno. 1992. Prosopis in Argentina: Initial results on cultivation in greenhouses and orchards, and pod quality for food or feed of five native Prosopis species of Córdoba province. En: Prosopis species. Aspects of their Value, Research and Development. Dutton, R. W. (Ed.) Proceedings of the Prosopis Symposium, Centre for Overseas Research and Development. University of Durham, UK. pp.145156.

Giménez, A.M., J.G. Moglia, N. Ríos, P. Hernández \& R. Gerez. 2016. Potencialidad de especies del genero Prosopis en la provincia de Santiago del Estero. Disponible en https://fcf.unse.edu.ar/eventos/2-jornadasforestales/pdfs/Potencialidad\%20de\%20Especies\%20d el\%20GE\%20Prosopis.pdf Último acceso mayo 2019.

Giménez, A.M., N. Rios, J.G. Moglia \& C. Lopéz. 1998. Leño y corteza de Prosopis alba Griseb., algarrobo blanco, Mimosaceae, en relación con algunas magnitudes dendrométricas. Bosque 19(2): 53-62.

Giménez, A.M., N. Rios, J.G. Moglia, P. Hernández \& S.J. Bravo. 2001. Evolución de magnitudes dendrometricas en funcion de la edad en Prosopis alba Griseb., algarrobo blanco, Mimosaceae. Revista Forestal Venezolana 45(1): 175-183.

Gómez-Sosa, E. \& R. Palacios. 1994. Prosopis L. En: Flora de San Juan - Vol. 1. Kiesling, R, (Ed.). Ed. Vázquez Mazzini, Buenos Aires, Argentina. pp: 264-272. González-Galán, A., A. Duarte-Corrêa, C. M. Patto De Abreu \& M.F. Piccolo-Barcelos. 2008. Caracterización química de la harina del fruto de Prosopis spp. procedente de Bolivia y Brasil. Archivos Latinoamericanos de Nutrición 58(3): 309-315.

Grados, N. \& G. Cruz. 1996. New approaches to industrialization of Algarrobo (Prosopis pallida) pods in Peru. En: P. Felker \& J. Moss (Eds.) Proceedings of the workshop: "Prosopis: semiarid fuelwood and forage tree, building consensus for the disenfranchised". Texas A\&M University, Washington, DC. 13-15 Marzo 1996. pp 3/2542.

Hieronymus, J. 1882. Plantas diafóricas. Editorial Atlántida. Buenos Aires, Argentina. 421 pp.

Hunziker, J., B.O. Saidman, C. Naranjo, R. Palacios, L. Poggio \& A. Burghardt. 1986. Hybridization and genetic variation of Argentine species of Prosopis. Forest Ecology and Management 16: 301-315.

Jarzinski, M., R. Velazco, D. Vergara, H. Álvarez \& C. Zunino. 2015. Aprovechamiento de los tintes vegetales del monte Chaqueño para su aplicación en artesanías e indumentaria. En: XXII Congreso Latinoamericano de Química Textil "Innovación Química, Tecnológica y Sostenibilidad". Peru, 19-21

Karlin, U.O. 1988. Argentina: Diversidad de Especies y Ambientes. En: Prosopis en Argentina. Primer Taller Internacional sobre Recurso Genético y Conservación de Germoplasma en Prosopis - FAO y Facultad de Ciencias Agrarias de la U.N. de Córdoba. 1: 1-14.

Karlin, U.O., R.O. Coirini, L. Catalan \& R. Zapata. 1997. Especies Arbóreas y Arbustivas para las Zonas Aridas y Semiáridas de América Latina - Prosopis alba. Disponible http://www.fao.org/tempref/GI/Reserved/FTP FaoRlc/ol $\mathrm{d} / \mathrm{redes} /$ sisag/arboles/Arg-p-al.htm Último acceso: mayo 2019.

Killian, S. 2012. Tolerancia a salinidad en Prosopis chilensis Moll Stuntz durante la germinación. Biología en Agronomía 2(2): 108-118. 
Lamarque, A., D. Maestri, N. Grosso, J. Zygadlo \& C. Guzmán. 1994. Proximate composition and seed lipid components of some Prosopis (Leguminosae) from Argentina. Journal of the Sciences of Food and Agriculture 66: 323-326.

Ledesma, T., G. De Bedia \& C. López. 2008. Productividad de Prosopis alba Griseb. en Santiago del Estero. Quebracho 15(1): 5-9.

Legume Phylogeny Working Group. 2017. A new subfamily classification of the Leguminosae based on a taxonomically comprehensive phylogeny. Taxon 66(1): 44-77.

López, C. 2005. Evaluación de la Variación Genética de Especies del Género Prosopis de la Región Chaqueña Argentina para su Conservación y Mejoramiento. En: Mejores Árboles para más Forestadores. Norberto, C. (Ed.). Secretaria de Agricultura, Ganadería y Pesca, Buenos Aires, Argentina. pp.195- 203.

López-Lauenstein, D., C. Luna \& A. Verga. 2010. Respuesta al estrés hídrico en dos grupos morfológicos de Prosopis alba. En: XXVIII Reunión Argentina de Fisiología Vegetal. La Plata, Argentina. Septiembre de 2010.

López-Lauenstein D., C. Vega, C. Luna, M. Sagadin, M. Melchiorre, E. Pozzi, C. Salto, G.P.J. Oberschelp, S. Torales, M. Pomponio, S. Kees, L. Chavez-Diaz, C. Gómez \& A. Verga. 2015. Subprograma Prosopis. En: Domesticación y mejoramiento de especies forestales. Ministerio de Agricultura Ganadería y Pesca, Buenos Aires, Argentina. pp. 113-135.

Macías, S., M. Binaghi, A. Zuleta, P. Ronayne De Ferrer, K. Costa \& S. Generoso. 2013. Desarrollo de galletas con sustitución parcial de harina de trigo con harina de algarroba (Prosopis alba) y avena para planes sociales. Revista Venezolana de Ciencia y Tecnología de Alimentos 4(2): 170-188.

Macías-Rodríguez, E. \& J. Usca-Méndez. 2017. Use of algarrobo flour (Prosopis pallida) in the feeding of rabbits in growth, fattening. Ciencia Unemi 10(22): 105-110.

Margalef, M.I., S.L. Tóffoli, V.E. Burgos, A. Campos, G.A. Valdez-Clinis \& M.J. Jiménez. 2012. Algarroba negra (Prosopis nigra): caracterización físicoquímica y elaboración de productos dietéticos. Revista de la Facultad de Ciencias de la Salud - Universidad Nacional de Salta 1(2): 13-19.

Martínez-Crovetto, R. 1964. Estudios etnobotánicos. Nombres de plantas y su utilidad según los indios tobas del este del Chaco. Bonplandia 1: 279-333.

Meyer, D., R. Becker, M. R. Gumbmann, P. Vohra, H. Neukom \& M. Saunders. 1986. Processing, composition, nutritional evaluation, and utilization of Mesquite (Prosopis spp.) pods as a raw material for the food industry. Journal of Agricultural and Food Chemistry 34: 914-919.

Michela, F.J., S. Kees \& J. Skoko. 2015. Evaluación del crecimiento de plantaciones juveniles de algarrobo blanco (Prosopis alba Griseb.) en el centro oeste del chaco argentino. Temas Agrarios 20: 11-20.

Millán, M.P., M.V. López-Márquez \& A.N. Ramón. 2016. Obtención de goma de semillas de algarroba (Prosopis alba) y su utilización en formulaciones alimenticias. Diaeta 34(157): 28-33.

Ministerio de Agroindustria. $\mathrm{s} /$ fecha. Características de la región Parque Chaqueño. Disponible en http://forestoindustria.magyp.gob.ar/archivos/informacio n-por-region/parque-chaqueno.pdf. Último acceso: enero 2020.

Ministerio de Ambiente y Desarrollo Sustentable de la Nación Argentina. 2020. Infografía: Especies más utilizadas para la producción de rollizos por región forestal (período 2007-2016). Disponible, en https://bosques.ambiente.gob.ar/geomaps. Último acceso: enero 2020.

Mom, M.P. 2012. Caracterización estructural y propiedades funcionales de las harinas de los frutos de Prosopis alba Griseb., P. chilensis (Molina) Stuntz emend. Burkart y $P$. flexuosa DC. Desarrollo de un proceso de secado, molienda y mezcla para optimizar la calidad del producto. Tesis Doctoral área Ciencias Biológicas. Universidad de Buenos Aires, Facultad de Ciencias Exactas y Naturales. Argentina. 117 pp.

Ocampo, T.L. 2011. Ficha Técnica Sistemas Silvopastoriles. México: Secretaria de agricultura, ganadería, desarrollo rural, pesca y alimentación (SAGARPA), Subsecretaría de desarrollo rural Dirección general de apoyos para el desarrollo rural. México. 8 pp.

Ochoa de Cornelli, M.J. 1996. A review of the Social and Economic Opportunities for Prosopis (Algarrobo) in Argentina. En: P. Felker \& J. Moss (Eds.) Prosopis: Semiarid Fuelwood and Forage Tree Building Consensus for the Disenfranchised. U.S. National Academy of Sciences Building. Washington, DC. 13-15 Marzo 1996.

Palacios, R. \& M. Brizuela. 2005. Fabaceae, parte 13. Subfam. II. Mimosoideae, parte 4. Tribu VI. Mimoseae, parte B. Prosopis L. En: A. M. Anton \& F. O. Zuloaga (Eds.), Flora Fanerogámica Argentina - Vol. 92. pp.3- 25. Pasiecznik, N., P. Felker, P. Harris, L. Harsh, G. Cruz, J. Tewari, K. Cadoret \& L. Maldonado. 2001. The Prosopis juliflora - Prosopis pallida Complex: A Monograph. HDRA, Coventry, UK. 172 pp.

Pasiecznik, N., P. Harris \& S. Smith. 2004. Identifying tropical Prosopis species: a field guide. HDRA, Coventry, UK. 30 pp.

Pozzi-Tay, E. 2016. Simbiosis en Prosopis alba como estrategia para la mejora de su tolerancia a estrés hídrico. Tesis de Grado. Universidad Nacional de Córdoba. Facultad de Ciencias Exactas, Físicas y Naturales. Carrera de Ciencias Biológicas. 28 pp.

Prokopiuk, D.B. 2004. Sucedáneo del café a partir de algarroba (Prosopis alba Griseb.). Tesis de Doctorado. Universidad Politécnica de Valencia, Departamento de Alimentos. España. 104 pp.

Prokopiuk, D.B., G. Cruz, N. Grados, O. Garro \& A. Chiralt. 2000. Estudio comparativo entre frutos de Prosopis alba y Prosopis pallida. Multequina 9:35-45.

Roser, L.G., L.I. Ferreyra, M. Ewens, J.C. Vilardi \& B.O. Saidman. 2013. Genetic and morphometric characterization of clones of Prosopis alba, Algarobia, selected for salt tolerance. Tree Genetics \& Genomes 10(2): 383-397.

Rozycki, V., C. Baigorria, C. Bernardi, M. Zannier \& C. Osella. 1998. Optimización de Molienda de Frutos de Prosopis alba y Ensayos de Panificación. Simposio Iberoamericano sobre Proteínas para Alimentos. Buenos Aires. pp.113- 127.

Rusconi, C. 1962. Poblaciones Pre y Post Hispánicas de Mendoza. Mendoza. Vol. 1: Etnografía: 
Antecedentes; El ocaso Huarpe; Número de aborígenes; Medios de locomoción; artes y artesanías; Utensilios, vestimenta y alimentación; ritos; música; motivos decorativos. Imprenta Oficial. Mendoza, Argentina. 750 pp.

Salto, C. 2011. Variación genética en progenies de polinización abierta de Prosopis alba Griseb. de la región Chaqueña. Tesis presentada como requisito para la obtención del Grado Académico de magister en genética vegetal. Maestría en Genética Vegetal - Área de mejoramiento genético. Universidad Nacional de Rosario. 97 pp.

Salto, C. S. \& A. M. Lupi. 2019. Avances en el conocimiento y tecnologías productivas de especies arbóreas nativas de Argentina. Disponible en https://inta.gob.ar/sites/default/files/avances_en_el_con ocimiento_y_tecnologias_productivas_de_especies_arb oreas_nativas_de_argentina_0.pdf. Último acceso: enero 2020.

Saravia-Sánchez, J. 2012. Una alternativa de recría de vaquillonas en un sistema silvopastoril en bosque nativo del chaco semiárido de la provincia de Santiago del Estero. Tesis presentada como requisito para la obtención del Grado Académico de Especialista en Alimentación de Bovinos. Universidad Nacional de Córdoba. Facultad de Ciencias Agropecuarias. Escuela para Graduados. 26 pp.

Sciammaro, L.P. 2015. Caracterización fisicoquímica de vainas y harinas de algarrobo. Tesis Doctoral. Universidad Nacional de La Plata. Facultad de Ciencias Exactas. $273 \mathrm{pp}$.

Secretaría de Gobierno de Ambiente y Desarrollo Sustentable. 2019. Anuario de Estadística Forestal 2017-2018. Disponible

https://www.argentina.gob.ar/ambiente/tierra/bosquessuelos/manejo-sustentable-bosques/programa-

nacional-estadistica-forestal. Último acceso: enero 2020.

Secretaría de Planificación y Evaluación de Resultados de la Provincia del Chaco. 2010. El Chaco en cifras - $2010 . \quad$ Disponible en http://estadisticas.chaco.gov.ar/wp-

content/uploads/2017/10/Chaco-en-cifras-2010.pdf.

Último acceso: enero 2020.

Secretaría de Política Económica - Programa de Competitividad del Norte Grande. 2009. Plan de Competitividad del Conglomerado Productivo de Muebles de Madera del Chaco. Ministerio de Economía y Finanzas Públicas - Gobierno del Chaco. 52 pp.

Singleton, V. 1981. Naturally occurring food toxicants: phenolic substances of plant origin common in foods. Advances in Food Research 27:149- 242.

Tagliamonte, C., U. Martinez-Ortiz, L. Longo \& S. DalPont. 2014. El servicio de provisión de forraje en el bosque nativo chaqueño: Una aproximación al análisis económico. Agronomía \& ambiente - Revista de la Facultad de Agronomía UBA 34(1-2): 37-46.
Taleisnik, E. \& D. López-Lauenstein. 2011. Leñosas perennes para ambientes afectados por salinidad. Una sinopsis de la contribución argentina a este tema. Ecología Austral 21: 3-14.

Tortorelli, L.A. 2009. Maderas y bosques argentinos Segunda edición actualizada. Orientación Gráfica Editora, Buenos Aires, Argentina. 515 pp.

Traskauskas, C.P., G.S. Glibota \& G.E. Camprubi. 2001. Comparación de productos derivados de la chaucha de algarrobo con sus sustitutos de mercado como evaluación inicial para un proyecto productivo. En: Reunión de Comunicaciones Científicas y Tecnológicas, SECYT, UNNE. Corrientes, Octubre de 2001. http://www.unne.edu.ar/unnevieja/Web/cyt/cyt/2001/7Tecnologicas/T-069.pdf

Venier, P., A. Cosacov, D. López-Lauenstein, C. Vega \& A. Verga. 2013. Impacto del cambio climático sobre la distribución de Prosopis hassleri y $P$. alba en la región Chaqueña. Produccion forestal 7(3): 35-38.

Verga, A. 2005. Recursos genéticos, mejoramiento y conservación de especies del género Prosopis. En: C. A. Norverto (Ed.), Mejores árboles para más forestadores: el programa de producción de material de propagación mejorado y el mejoramiento genético en el Proyecto Forestal de Desarrollo. Secretaria de Agricultura, Ganadería y Pesca. Buenos Aires, Argentina. pp. 205222.

Verga, A., D. López-Lauenstein, C. López, M. Navall, J. Joseau, C. Gómez, O. Royo, W. Degano \& M. Marcó. 2009. Caracterización morfológica de los algarrobos (Prosopis sp.) en las regiones fitogeográficas Chaqueña y Espinal norte de Argentina. Quebracho 17(1,2): 31-40.

Villagra, P. 2000. Aspectos ecológicos de los algarrobales argentinos. Multequina 9(2): 35-51.

Villagra, P. \& M.S. Morales. 2003. Dendroecology of Prosopis woodlands in the Argentine arid zone. IANIGLA 1973-2002: 53-57.

Villagra, P., A. Vilela, C. Giordano \& J. Álvarez, J. 2010. Ecophysiology of Prosopis species from the arid lands of Argentina: What do we know about adaptation to stressful environments?. En: K. G. Ramawat (Ed.), Desert Plants. Biology and Biotechnology. SpringerVerlag, Berlin-Heidelberg. pp.322- 354.

Zuleta, A., M.J. Binaghi, C.B. Greco, C. Aguirre, L. De La Casa, C. Tadini \& P.A. Ronayne De Ferrer. 2012. Diseño de panes funcionales a base de harinas no tradicionales. Revista chilena de nutrición 39(3): 58-64.

Zurloaga, F.O., O. Morrone \& M.J. Belgrano. 2008. Flora de la República Argentina. Catálogo de plantas vasculares del Cono Sur (Argentina, Sur de Brasil, Chile, Paraguay y Uruguay). Missouri Botanical Garden Press. St. Louis. 3486 pp. 